La revista Corga (2010-2016): análisis, trayectoria e influencia de una revista para promover la lengua gallega y el patrimonio material e inmaterial de $\mathrm{O}$ Corgo

Montserrat Doucet ${ }^{1}$

Recibido: 12 de abril de 2019 / Aceptado: 27 de novembro de 2019

Resumen. En marzo de 2009 se constituye dentro del ámbito rural gallego el Centro de Estudios de O Corgo como una asociación sin ánimo de lucro, que entre otros objetivos propone la defensa de la cultura y la lengua de Galicia así como diferentes estudios multidisciplinares del patrimonio natural, material e inmaterial del concello de $\mathrm{O}$ Corgo. La revista anual Corga es uno de los instrumentos que posibilita la consecución de dichos objetivos. Trataremos de analizar a lo largo de sus seis números como ha reflejado las inquietudes culturales de una parte del pueblo gallego y como ha influido en su entorno.

Palabras clave: gallego rural; Asociación Arumes; O Corgo; patrimonio material; patrimonio inmaterial.

\title{
[gal] A revista Corga (2010-2016): análise, traxectoria e influencia dunha revista para promover a lingua galega e o patrimonio material e inmaterial do Corgo
}

Resumo. En marzo de 2009 constitúese dentro do ámbito rural galego o Centro de Estudos do Corgo como unha asociación sen ánimo de lucro, que entre outros obxectivos propón a defensa da cultura e a lingua de Galicia así como diferentes estudos multidisciplinares do patrimonio natural, material e inmaterial do concello do Corgo. A revista anual Corga é un dos instrumentos que posibilita a consecución dos devanditos obxectivos. Trataremos de analizar ao longo dos seus seis números como reflectiu as inquietudes culturais dunha parte do pobo galego e como influíu na súa contorna.

Palabras chave: galego rural; Asociación Arumes; O Corgo; patrimonio material; patrimonio inmaterial.

[en] Corga Magazine (2010-2016): Analysis, Trajectory and Influence of a Magazine to Promote the Galician Language and the Tangible and Intangible Heritage of O Corgo

\begin{abstract}
In March 2009, Corgo Studies Center is constituted within the Galician rural environment as a non-profit association among other objectives proposed defense of the culture and the language of Galicia as well as different multidisciplinary studies of the natural heritage, material and immaterial of the concello de O Corgo. The annual magazine Corga is one of the instruments that makes it possible to achieve these objectives. We will try to analyze throughout their six numbers as it reflected the cultural part of the Galician people concerns and as influenced in their environment. Keywords: Galician Rural; Arumes Association; O Corgo; Tangible Heritage; Intangible Heritage.

Sumario. 1. Introducción. 2. El Centro de Estudios de O Corgo. 3. La lengua gallega en Corga. 4. El patrimonio material. 5. El patrimonio inmaterial. 6. La repercusión de la revista en la vida rural de O Corgo. 7. A modo de conclusión. 8. Referencias bibliográficas.

Como citar: Doucet, M. (2020): “La revista Corga (2010-2016): análisis, trayectoria e influencia de una revista para promover la lengua gallega y el patrimonio material e inmaterial de O Corgo", en Madrygal. Revista de Estudios Gallegos 23 Núm. Especial, pp. 89-95.
\end{abstract}

\footnotetext{
1 Universidad Complutense de Madrid. Departamento de Estudios Románicos, Franceses, Italianos y Traducción.
} Correo-e: doucet.montserrat19@gmail.com; https://orcid.org/0000-0003-0834-2091. 


\section{Introducción}

En O Corgo, población situada al sur de Lugo de hábitat muy disperso, surge en el otoño de 2005 una asociación denominada Arumes que apuesta por la educación en el medio rural gallego. En la Revista Galega de Educación, núm. 52 (febreiro 2012), leemos lo siguiente:

arumes é o nome das follas do piñeiro que ademais de aparecer no himno de Galiza, non deixan de ser a acícula da variedade forestal máis estendida no noso concello. Arumes créase como un espazo de formación, aprendizaxe, reflexión e construción conxunta da realidade galega no noso concello. Durante estes seis anos de traxectoria leváronse a cabo actividades que acollen ámbitos do máis diversos. Todas e cada unha delas amosan unha parte da sociedade educadora no que a asociación pretende facerse un oco. Deste xeito, Arumes convértese nunha entidade que participa na educación en valores, tema de gran impacto social e transversal en canto á educación e fomento. (Outeiro Iglesias e Páramo Iglesias 2012: 83)

La Asociación Cultural Arumes de O Corgo (http://arumescorgo.blogspot.com/) promoverá el conocimiento del patrimonio, la naturaleza y la etnografía con el trabajo de sus socios, la ayuda de instituciones públicas y la colaboración de asociaciones de la comarca. Cuentacuentos, jornadas sobre artesanía, patrimonio, oficios artesanales y cine, excursiones para conocer el patrimonio de la comarca, certámenes deportivos y el certamen literario "Terras de Chamoso" para promover la creación en lengua gallega, serán algunas de sus actividades. Asimismo, reivindica la lengua gallega como queda expresamente manifestado en su acta número 10 :

A vicepresidenta da Asociación fai lectura dun manifesto na defensa da lingua galega ante as propostas do futuro executivo galego en materia lingüística. A maiores realízase unha recollida de sinaturas entre os socios co fin de avalar o devandito manifesto e envialo ao Sr. Presidente da Xunta de Galicia. Apróbase por unanimidade.

O secretario da Asociación propón a adhesión de Arumes na Asociación PuntoGal, que é unha iniciativa que pretende conseguir para a lingua e a cultura galegas un dominio propio en internet e, a través del, unha maior visibilidade e presenza do pobo galego na rede e no mundo. Apróbase por unanimidade. (Acta $n^{\circ}$ 10, 2009: 3)

De la asociación Arumes partirá la propuesta de constituir "baixo o paraugas da Asociación
Cultural Arumes do Corgo, outra Asociación denominada Centro de Estudos do Corgo coa idea de investigar (e espallar) os distintos aspectos culturais do noso concello e da nosa área de influencia" (Acta $n^{\circ}$ 10, 2009: 3). Dicha propuesta será aprobada por unanimidad.

\section{El Centro de Estudios de O Corgo y la re- vista Corga}

Según consta en el acta fundacional del Centro de Estudios de O Corgo el 28 de marzo de 2009 se reunen varios miembros de la asociación Arumes: $\mathrm{M}^{\mathrm{a}}$ Consuelo Vázquez, Manuel Fernández López, Roberto Rodríguez Páramo, Josefa González Díaz, Mario Otero Iglesias Manuel Marqués Reija y Jorge José Rodríguez Lozano; todos ellos vecinos de Corgo que acuerdan:

1. Constituír unha asociación sen ánimo de lucro, ao abeiro da vixente Lei orgánica 1/2002, de 22 de marzo, reguladora do dereito de asociación, denominada Centro de Estudos do Corgo, que desenvolverá principalmente as súas actividades no ámbito do concello do Corgo e terá o seu domicilio nas Antigas Escolas do Corgo s/n, C.P. 27.163, concello do Corgo, os seus fins principais son:

a) Promover diferentes estudos (patrimonio natural, material e inmaterial, arte, lingua e literatura, xeografía e historia, economía, sociedade e educación...) sobre o concello do Corgo.

b) A divulgación dos estudos realizados entre os habitantes do concello.

c) A formación dos/as socios/as e dos/as corgueses/as sobre as áreas de estudo a nivel supralocal.

d) A defensa en xeral da cultura e da lingua de Galiza. (Acta fundacional, 2009: 1-2)

Para llevar a cabo estos fines se acuerda también organizar diversas actividades entre las que se encuentra la creación de una revista "anual (por escrito) con artigos sobre O Corgo" (Acta fundacional, 2009: 2).

Los socios fundadores del CEC son los mismos que constituirán el consejo editorial y la junta directiva de la revista: $\mathrm{M}^{\mathrm{a}}$ Consuelo Vázquez Castro (presidenta), Manuel Fernández López "Lolo" (vicepresidente), Roberto Rodríguez Páramo (secretario), Josefa González Díaz (tesorera) y Mario Outeiro Iglesias, Xurxo Xosé Rodríguez Lozano y Manuel Marqués Reija (vocales).

De principio a fin de la revista la presidencia la ostentará $\mathrm{M}^{\mathrm{a}}$ Consuelo Vázquez Castro; no 
así la vicepresidencia, que a partir del número 3 la ejercerá Josefa González Díaz, tesorera en los números 1 y 2 ; como tesorero durante los años 2012 a 2015, números 4 y 5, figura Xosé Enrique Casanova, volviendo a recaer en Josefa González Díaz durante 2016, número 6, ejerciendo la vicepresidencia durante ese año Mario Outeiro Iglesias. La secretaría recae en Roberto Rodríguez Páramo durante los años 2010 y 2011, números 1 y 2, y en 2012-2015 en Xurxo José Rodríguez Lozano, número 3, 4 y 5 . En cuanto a los vocales, figuran Mario Outeiro Iglesias, Xurxo Xosé Rodríguez Lozano y Manuel Marqués Reija en los números 1 y 2, años 2010-2011; Manuel Fernández López "Lolo", Xosé Manuel Castro Castedo y Roberto Rodríguez Páramo en los números 3, 4, 5 y 6, años 2012-2016.

La revista cuenta con comité científico, asesoría lingüística y asesoría TIC. El comité científico está integrado por los mismos miembros a lo largo de los seis números de Corga: Felipe Arias Vilas, Javier Gómez Vila, Xosé Manuel Castro Castedo, Rubén Ruibal Armesto, Ana Cabana Iglesia, Emilio Carral Vilariño, Daniel López Vispo, Xoán Ramiro Cuba Rodríguez, Antonio Reigosa Carreiras, Xabier Bruña García, Felipe-Senén López Gómez, Manuel Lamas Castro y Adolfo de Abel Vilela; en cuanto a la asesoría lingüística, constan varios colaboradores a lo largo de la historia de la revista: Xabier Cordal Fustes, número 1; Xosé Manuel Becerra Pardo, números 2, 3, 4, 5; y Noelia Arias Gómez, número 6. La asesoría TIC estará a cargo de Mario Outeiro Iglesias, números 1, 2; y Josefa González Díaz, números 1, 2, 3, 4, 5, 6 .

En cuanto a su estructura interna, el esquema de organización de la Asociación es el siguiente tal y como se refleja en el documento "E nace o CEC" (Corga 2010: 4):

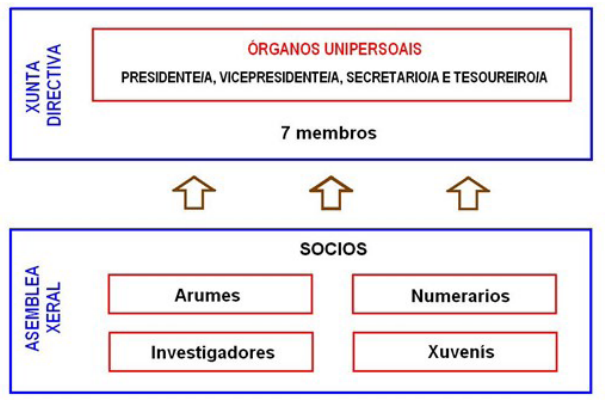

La edición de la revista corre a cargo del Centro de Estudos do Corgo (CEC) con ayuda económica institucional; se publicaron seis números entre 2010 y 2016. Además de los artículos se incluyen en todos los números la memoria de las actividades de Arumes durante ese año y boletines con instrucciones para la entrega de artículos, de inscripción para socios, de cesión de derechos de autor, de autorización familiar para menores de edad; también se incluyen las normas de publicación.

O Centro de Estudos do Corgo, a través desta e doutras iniciativas, pretende potenciar, fomentar, divulgar, defender a cultura en xeral de Galiza e a lingua en particular, centrados no noso ámbito de actuación, que é O Corgo, cunha proxección aberta cara ao futuro, sen esquecernos o noso pasado e os vencellos comúns que nos forman como país.

Non debemos esquecer que para que sexa posíbel esta publicación contamos coa colaboración inestimábel de persoas e institucións. $\mathrm{O}$ noso especial agradecemento ao Vicepresidente Primeiro da Deputación Provincial de Lugo, Antón Bao Abelleira, pola súa dedicación e implicación persoal con este e outros proxectos, así como á institución que representa polo financiamiento económico.

Corga se publica ininterrumpidamente entre 2010 y 2016, pero al día de hoy aún no ha visto la luz el número 7 en el que aún se sigue trabajando.

Son muchas las áreas que se abordan en la revista: lengua gallega, patrimonio material e inmaterial, emigración, estudios histórico económicos, etnografía, botánica o personajes importantes en la historia de O Corgo como Ánxel Fole. Analizaremos los tres primeros por ser los más recurrentes a lo largo de la vida de la revista. Es interesante el monográfico que se dedica a uno de los miembros de más peso tanto en Arumes como en el CEC, tras su muerte en un incendio forestal: el investigador Xosé Enrique Casanova ${ }^{3}$.

\section{La lengua gallega en Corga}

Ya se recoge en el acta 10 de la asociación Arumes la importancia que tendrá el gallego para la difusión y concreción de sus propuestas como hemos visto en el punto 1 de este trabajo.

En el editorial de Corga, número 6, pág. 3 podemos leer lo que supuso este socio para el CEC y la revista: "O ano 2015 foi especialmente triste para familia CEC-Arumes polo falecemento nun incendio forestal, do noso compañeiro Xosé Henrique Casanova Sánchez. Quique Casanova, como era coñecido na nosa contorna, sería socio numerario do Centro de Estudos de Corgo dende o primeiro momento e na renovación da Xunta Directiva efectuada o 5 
Asimismo, en el acta fundacional del CEC se subraya la importancia que tendrá la lengua gallega tanto en la asociación como en sus actividades, recogiéndose todo esto en su artículo sexto:

O idioma oficial da asociación será o galego. Farase uso deste nas asembleas, actas e documentos da asociación, así como en calquera tipo de publicidade ou comunicación pública. Considerarase tamén o seu uso en todas as actividades nas que sexa posíbel. O obxectivo é contribuír á plena normalización da lingua galega ${ }^{3}$.

La revista se publica íntegramente en lengua gallega y cuenta con asesoría lingüístca en todos y cada uno de sus números: Mario Outeiro Iglesias (número 1), Xosé Manuel Becerra Pardo (números 2, 3, 4, 5 y 6).

Tres son los estudios respecto a la lengua gallega en $\mathrm{O}$ Corgo que se publican a lo largo de estos seis números de la revista.

En el número 1 aparece un trabajo titulado "A microtoponimia do concello do Corgo" (6 pp.), de la autoría de Alejandra Pita Pardo, licenciada en Filología gallega e inglesa, en el que se nos ofrece una abundante cantidad de microtopónimos (más de 5.000) clasificados exhaustivamente, como en el caso de los orónimos: "(a) os que indican elevación do terreo; (b) os que indican depresión do terreo; (c) os que indican una vertente do terreo; (d) os que indican un chairo do terreo" (Corga 2010: 70).

En el número 2 se publica "O galego en Queizán. Vocalismo, consonantismo e fonética sintáctica" (12 pp.), por Xosé Manuel Castro Castedo, profesor de Filología hispánica por la Universidad de Santiago de Compostela y profesor de Enseñanza Secundaria. En este trabajo el autor analiza algunas de las características lingüísticas del habla de la parroquia de Queizán a partir de textos orales de una decena de vecinos y vecinas recogidos en grabaciones de mediados de la década de los 80 :

O estudo céntrase nos fenómenos fonolóxicos, aínda que se comentan algunhas variantes morfolóxicas, que afectan ao número dos substantivos ou ao sufixo diminutivo -iño e que teñen moita repercusión na dialectoloxía galega. A fonética sintáctica, tradicionalmente bastante esquecida nas pescudas lingüísticas, constitúe un aspecto importante no ámbito da lingua oral; porque os falantes non emitimos sons illados, senón que encadeamos palabras en frases. Polo tanto, estas concorrencias provocan a perda de fonemas e dun xeito especial no vocalismo átono. (Corga 2011: 6)

De este último autor es el artículo publicado en el número 3: "Léxico no Galego de Queizán" (9 pp.). En dicho trabajo, Xosé Manuel Castro Castedo analiza algunas particularidades del léxico coloquial oral partiendo de los datos de grabaciones recogidas por él mismo 25 años atrás y de su propia experiencia como hablante natural de dicha feligresía: las interferencias lingüísticas (castellanismos fonéticos con funcionalidad expresiva y castellanismos plenos) y algunos dialectalismos, clasificados en campos semánticos (tiempo, espacio, agricultura, el pan, las plantas, los animales).

\section{El patrimonio material}

La importancia de este aspecto para Corga lo apreciamos desde el mismo momento de la fundación del CEC al escoger como logotipo de la asociación el emblemático puente de Neira. El primer número de la revista también dedicará su portada a este monumento patrimonial exhibiendo una fotografía del mismo. Los motivos para escoger este motivo son fundamentalmente históricos:

O logotipo escollido representa a Ponte de Neira. Está situada no río Neira, $3,5 \mathrm{~km}$ río abaixo da desembocadura do Tórdea. Nese punto o río

de maio de 2012 pasaría a formar parte da mesma coas función de tesoureiro. O seu amor polo Corgo, pese a nacer en Lugo, e as súas inquedanzas no eido cultural e da natureza quedaron patentes nos seus tres artigos presentados, e polo tanto publicados, na revista Corga. Estes foron "Fórmulas de propiedade comunal dos montes do Corgo", publicado na revista Corga n $^{\circ}$ 1, "A Fervenza Reserva da Biosfera", publicado na Corga $\mathrm{n}^{\mathrm{o}} 3$ e "Pontes Históricas do Corgo, na $\mathrm{n}^{\circ}$ 4. Polo tanto, ao presentar tres traballos, Quique convertiríase no segundo socio Investigador do Centro de Estudos do Corgo. Sirva esta nova revista, un extenso artigo monográfico sobre a súa biografía, de homenaxe a unha persoa dunha extraordinaria valía que foi coñecido en toda a comarca lucense pola súa profesionalidade como axente forestal, polo seu activismo político e sindical, por ser impulsor de varias asociación do ámbito cultural e deportivo e por ser concellerio do BNG no Corgo durante 16 anos. Unha persoa honrada e xenerosa que loitou toda a súa vida pola xustiza social e a liberación do pobo galego".

3 Centro de Estudos de Corgo, Estatutos, capítulo I, "Da Asociación en Xeral". 
fai de división administrativa entre o concello do Corgo (Cela) e o do Páramo (Vilarmosteiro). Por ela pasa a estrada Lugo-Portomarín (pasando polo Páramo). É das pontes máis importantes do Sur de Lugo. Construída no s. XIII-XIV está ligada á primitiva vía de comunicación Sarria-Lugo. Xa aparecía nos antigos mapas de Galiza, como o de F. Fer. Ojea de 1598 ou no "Gallaecia Regnum” de 1600. Na idade media era o único vial que comunicaba o camiño de Santiago con Lugo. Hai un dato que confirma a anterior afirmación, pois na viaxe de regreso dos Reis Católicos á corte, logo da súa visita a Galicia no outono de 1486, saen de Lugo e diríxense a Ponferrada por Sarria e Triacastela, pasando tamén moi probabelmente pola ponte de Neira. Consta de catro vans de sillería de medio punto de luces: 11,20-11,20-8,00-7,00 m. (Corga 2011: 2)

Diversos y abundantes son los artículos dedicados al estudio del patrimonio material. En el número 1 de Corga se dedican dos artículos a este tema: un "Estudo das sepulturas antropoides altomedievales" (12 pp.) por Mario Outeiro Iglesias, ingeniero técnico industrial e ingeniero químico, profesor de secundaria; y un artículo sobre "A vía romana no concello de Corgo" (9 pp.), por Javier Gómez Vila, doctor en arqueología antigua y profesor de enseñanza secundaria.

En el número 2 encontramos tres estudios: "O camino real de acceso a Galicia e a súa pegada nas terras do Corgo" (13 pp.) por Javier Gómez Vila; "Catalogación de construccións rurais tradicionais. Os hórreos no concello do Corgo" (13 pp.) por Juan Ortiz Sanz y Miguel Ángel Piñeiro Gómez, ambos ingenieros agrónomos y el primero profesor titular en la Universidad de Santiago de Compostela; "O pazo do Corgo" (11 pp.) por Jesús Abuín Arias, estudioso de O Corgo y autor de varios libros sobre este ayuntamiento, y Josefa González Díaz, diplomada en Relaciones laborales y licenciada en Humanidades, funcionaria de la Universidad de Santiago de Compostela.

En el número 3 se publican tres artículos: "Cruceiros e cruces do Concello do Corgo" (6 pp.) por Fernando Arribas Arias, responsable de difusión de la Rede Museística de Lugo y profesor de humanidades; "Os escudos no concello do Corgo" (12 pp.) por Jesús Abuín Arias y Josefa González Díaz, ya citados; "A ferventa. Reserva da Biosfera” (10 pp.) por Xosé
Enrique Casanova Sánchez, agente facultativo medioambiental de la Consejería del Medio Rural y Marino.

Cuatro son los artículos que se publican en el número 4: "O relevo de $\mathrm{O}$ Corgo. Un exemplo da plástica provincial romana no Museo de Lugo"(7 pp.) por Aurelia Balseiro García, museóloga y directora del Museo Provincial de Lugo; "Pontes históricas do Corgo" (7 pp.) por $\mathrm{X}$. Enrique Casanova Sánchez; "Contos, pontes e pousadas do camiño real de acceso a Galicia” (12 pp.) por Javier Gómez Vila, doctor en Arqueología antigua, profesor de enseñanza secundaria; "Santuario do Santo Anxo de Anseáns (O Corgo)" (16 ppp.) por José Manuel Blanco Prado.

En Corga número 5 aparecen tres nuevos trabajos: "A ara votiva de Adai e a promesa a Mercurio" (16 pp.) por Carolina Casal Chico, conservadora del Museo Diocesano Catedralicio de Lugo; "Os castros da Terra do Corgo" (16 pp.) por Javier Gómez Vila; "Outra mostra da plástica galaico-romana no Corgo: os pés de Bergazo" (12 pp.) por Aurelia Balseiro García.

\section{Patrimonio inmaterial}

La Unesco define el patrimonio oral e inmaterial como "el conjunto de creaciones basadas en la tradición de una comunidad cultural expresada por un grupo". Lengua, literatura, música y danza, juegos y deportes, tradiciones culinarias, los rituales y mitologías, conocimientos y usos relacionados con el universo, los conocimientos técnicos relacionados con la artesanía y los espacios culturales se encuentran entre las muchas formas de patrimonio inmaterial. El patrimonio inmaterial es visto como un depósito de la diversidad cultural, y la expresión creativa, así como una fuerza motriz para las culturas vivas. Como se puede ser vulnerable a las fuerzas de la globalización, la transformación social y la intolerancia, la Unesco alienta a las comunidades a identificar, documentar, proteger, promover y revitalizar ese patrimonio ${ }^{4}$.

Tanto la Asociación Arumes como después el CEC abogan por la recuperación y transmisión de dicho patrimonio. En el número 2 de la revista aparecen dos publicaciones que hacen referencia a este aspecto: "Recollida do patrimonio inmaterial no Corgo: unha materia

14 https://es.wikipedia.org/wiki/Patrimonio_cultural_inmaterial_de_la_Humanidad. 
pendiente" (14 pp.) por Mario Outeiro Iglesias, quien propone la recogida de este material en el concejo de una manera sistemática y científica, cuyo "fin é honroso, devolvérllelo aos veciños dun xeito didáctico e ordenado co fin de mellorar a autoestima do rural e intentar que os engrenaxes transmisores de tan rico saber sigan avanzando no tempo" (Corga 2011: 82). Por otra parte, el artículo titulado "O traballo compilador de Francisco Vázquez Saco: as oracións de elaboración do pan e outras tradicións orais do Corgo" (16 pp.) por José Luis Garrosa Gude, licenciado en Filología alemana y en Derecho, profesor de secundaria en Madrid.

Otros dos artículos sobre patrimonio inmaterial se publicarán en el número 3: "Etnorituais e ofrendas relixiosas a nivel popular en dúas comunidades do Corgo (Lugo): Aseán e Castrillóns (1)" (10 pp.) por José Manuel Blanco Prado; y "Os teleclubs do concello do Corgo (1967-1984): unha achega inicial” (12 pp.) por Juan José García Rodicio, posgraduado en Servicios Culturales

Respecto a los números 4 y 5 de Corga, también podrían incluirse aquí los artículos reseñados en el apartado anterior: "Contos, pontes e pousadas do camiño real de acceso a Galicia" (núm. 4); y "De feira en feira. Hoxe imos ao Corgo" (núm. 5, 10 pp.) por Xema Sanxurxo Rodríguez, licenciada en Filología gallega con máster en Servizos Culturais.

En el número 6 se puede leer un estudio sobre una de las actividades tradicionales en el medio rural: "Análise etnohistórico do cultivo do liño no concello de O Corgo" (13 pp.) por José Manuel Blanco Prado y Manolo Fernández López "Lolo", doctor en Historia, profesor de enseñanza secundaria y ceramista, respectivamente.

\section{Los receptores}

Corga nace como un instrumento del CEC de difusión entre los habitantes de O Corgo a través de los trabajos publicados en la misma, por lo que los primeros receptores son los propios corgueses y corguesas:

O CEC naceu para que todos poidamos aprender sobre o noso concello a través dos variados traballos que os autores nos van presentando ano tras ano e que tratan temas tan diversos como a lingua, o patrimonio arquitectónico, a historia ou a etnografía. (Corga 2011: 3)

En el editorial del número 2 ya se hace constar el interés que ha despertado la revista y como este hecho consolida al CEC como institución cultural:

O Centro de Estudos do Corgo está a consolidarse no panorama cultural deste concello, grazas ao interese que espertou esta revista entre os corgueses e corguesas, así como noutras poboacións, polos excelentes traballos presentados no primeiro número, que afondaron no coñecemento do patrimonio sociocultural deste concello. (Corga 2011: 3)

Es también en este número donde se reconoce la labor de los lectores: "Por último, grazas a ti, lector, porque se liches esta moderna editorial é porque algo te une, como a nós, cunha terra apaixonante, O Corgo" (Corga 2011: 3).

La revista ya se planteó desde el inicio como algo necesario para la propia historia del pueblo de O Corgo y este objetivo no se abandonó a lo largo de los sucesivos números; así, en el editorial del número 3 se recuerda en los siguientes términos:

A historia dos pobos precisa de fontes nas que se mirar e, sen dúbida, a do Corgo ten nesta publicación unha fonte de auga fresca na que poidan beber as futuras xeracións. Sensibilizar e implicar ás distintas institucións para a difusión dos traballos que van aparecendo na revista é outro dos obxectivos que nos fixamos. (Corga 2014: 3)

En el editorial de este mismo número se notifica que "Así, pouquiño a pouco, viron a luz na CORGA 21 estudos individuais ou colectivos que propiciaron outras tantas conferencias divulgativas no Centro Sociocultural". Por tanto, la labor de difusión de la revista logró despertar suficiente interés entre la población para organizar conferencias de carácter divulgativo, y lo que no es menos importante: lograr captar el interés de las instituciones y su colaboración económica: "Agradecemos tamén á Area de Cultura da Deputación de Lugo a sensibilidade que veu demostrando hacia este proxecto desde os seus inicios e polo financiamento que o fai posible" (Corga 2012: 3).

En el número 4 se habla de colaboraciones de ultramar, lo que nos indica que la revista ya es conocida al otro lado del Atlántico:

apreciará colaboracións que veñen dende o outro lado do Atlántico realizadas por persoas con nostalxia e aprezo ás súas orixes. (...) Non queda máis que animar aos lectores a que sigan descubrindo a través destas páxinas, todos os valores que o Corgo atesoura, defendendo sempre a 
través do idioma que nos une, a nosa cultura e o noso territorio (Corga 2013: 3).

\section{A modo de conclusión}

La revista Corga nace de un proyecto educativo multidisciplinar, popular y en un entorno rural promovida por personas que pertenecen al mundo de la cultura y la docencia en su mayor parte. El elemento aglutinador es su amor y pasión por fomentar, recuperar y expandir el rico patrimonio de $\mathrm{O}$ Corgo en todas sus vertientes.

La lengua gallega será una prioridad y el vehículo para transmitir los contenidos desde el comienzo. En este sentido, Corga cuenta, como se ha visto, con asesoría lingüística desde el primer número. La revista también recoge año tras año el resultado del certamen de creación literaria "Terras de Chamoso", promovido por la asociación Arumes y el CEC, y en el que participan escritores de toda Galicia.

Corga ha tenido una rápida y buena acogida por parte de las instituciones, cuya colaboración económica e institucional hace posible la publicación y sostenibilidad de la revista.
También es conocida y leída por suficiente gente, también al otro lado del Atlántico, como para organizar, entre otros eventos, seminarios divulgativos relacionados con los contenidos publicados en la revista.

La revista Corga, asimismo, contribuye a fortalecer los lazos de solidaridad entre sus miembros y lectores, tal como demuestra el artículo monográfico publicado en el número 6 sobre uno de sus colaboradores tras su fallecimiento en un incendio forestal. El hecho de que se haya interrumpido la publicación durante dos años, aunque se siga trabajando en el siguiente número, indica en parte la influencia de este hecho entre sus miembros.

Por último y en definitiva, cabe destacar el impacto positivo de O Corgo, considerada no como una simple comarca rural en apariencia dedicada a la ganadería y a las labores del campo, sino como un hábitat natural repleto de riquezas materiales e inmateriales, y un espacio humano de una gran inquietud cultural, lo que ha hecho posible un proyecto tan ambicioso como es la Asociación Arumes, el Centro de Estudos do Corgo y la propia revista Corga.

\section{Referencias bibliográficas}

Centro de Estudos do Corgo (2009): Acta Fundacional. O Corgo: Asociación Arumes, Centro de Estudos do Corgo.

Centro de Estudos do Corgo (2009): Acta $n^{\circ} 10$. O Corgo: Asociación Arumes, Centro de Estudos do Corgo.

Centro de Estudos do Corgo (2010-2016): Revista Anual do Centro de Estudios do Corgo (números 1-6). O Corgo: Asociación Arumes, Centro de Estudos do Corgo, https://www.concellodocorgo.com/index.php/ documentacion/documentacion/corga.html.

Outeiro Iglesias, Mario e María Beatriz Páramo Iglesias (2012): “A escola rural. O proxecto culturaleducativo Arumes do Corgo", Revista Galega de Educación 53, pp. 88-91, https://issuu.com/revistagalegadeeducacion/docs/rge_53_completa. 